\title{
Tecnologias e o ensino na área da Saúde
}

\section{Technology and education in the area of Health}

\author{
Arquimedes Pessoni ${ }^{1}$, Elias Goulart ${ }^{1}$ \\ 'Universidade Municipal de São Caetano do Sul (USCS) - São Caetano do Sul (SP), Brasil.
}

DOI: http://dx.doi.org/10.7322/abcshs.v40i3.807

\section{RESUMO}

O artigo de revisão versa sobre a mudança de configuração dada em sala de aula e fora dela a partir da adoção de redes sociais digitais como possibilidade de relacionamento entre alunos e professores. Os trabalhos consultados revelam uma tendência à adoção de novas formas de ensino e aprendizagem a partir da inclusão de tecnologia mediando os públicos envolvidos. Ressaltando trabalhos de sucesso e outros não muito felizes em que novas ferramentas foram adotadas por professores, as considerações apontam para a necessidade de adaptação por parte dos docentes para uma nova realidade de mercado em que o cliente, entendido aqui como aluno, traz consigo uma nova forma de aprender, inserindo a tecnologia como ferramenta necessária para que o conteúdo seja assimilado mais facilmente.

Palavras-chave: ensino; aprendizagem; rede social; redes de comunicação de computadores; docentes; estudantes.

\section{ABSTRACT}

The review focuses on the change of setting given in the classroom and beyond from the adoption of online social networks as a possibility of relationship between students and teachers. The works consulted show a tendency to adopt new forms of teaching and learning from the inclusion of mediating technology stakeholders involved. Emphasizing works of success and others not very happy in that new tools were adopted by teachers, the considerations point to the need for adaptation on the part of teachers to a new reality where the customer market, understood here as a student, brings with it a new way of learning by technology as a necessary tool for content to be assimilated more easily.

Keywords: teaching; learning; social networking; computer communication networks; faculty; students. 


\section{INTRODUÇÃO}

A reflexão apontada neste trabalho se contextualiza em amplo cenário, em parte já mapeado e noutra parte obscuro, inóspito e desafiador. Como ponto de partida, cita-se o pensamento do professor Mark J. Kittleson', que afirma: "There is no question that the future of health education (or probably any profession) lies in its ability to survive in the technological world". As tecnologias em geral e, em especial, as baseadas nos processos digitais, como a digitalização das informações, suas transformações por diversos softwares, os mecanismos de armazenagem, recuperação, transmissão e distribuição em escala global com custos ínfimos, potencializaram ao longo das últimas décadas uma revolução em todas as dimensões da vida humana.

Em termos educacionais, as discussões causam celeumas teórico-didático-prática-metodológicas, enfim, em todas as dimensões dos processos de ensino e aprendizagem. O professor Francisco Rüdiger ${ }^{2}$ postula a dicotomia nas relações com a tecnologia, caracterizando os perfis das pessoas como os tecnófilos, que se "apaixonam" pelos avanços tecnológicos e os adotam e buscam empregá-los para tirar deles os melhores proveitos, e aqueles outros que se horrorizam com a aventada alienação humana, desfiguração da pessoa causada pelas consequências maléficas das tecnologias na vida e nas relações humanas, enfim, os chamados tecnófobos.

$\mathrm{Na}$ área da Educação as análises também podem ser categorizadas de forma similar, contudo nosso enfoque ${ }^{3}$ tem sido que as tecnologias potencializam o ser humano, amplificando suas ações, seja no sentido positivo, com resultados benéficos para si e para a sociedade, ou no sentido negativo. O professor Nicholas Negroponte, em seu texto clássico denominado "A vida digital"4, propõe à imaginação que, se um cirurgião do século XIX fosse colocado em um centro cirúrgico atual, ele não conseguiria atuar, diante de todos os recursos tecnológicos disponíveis e necessários; contudo, se um professor da mesma época fosse colocado em uma sala de aula atual, ele conseguiria ministrar sua aula.

O principal objetivo dessa reflexão é discutir alguns aspectos julgados relevantes desse contexto, restrita basicamente pelo espaço disponível, no sentido de contribuir para que o melhor das tecnologias digitais possa ser apropriado pelos atores educacionais em prol do estudante, centro e último beneficiário de todo o esforço educativo.

Inicialmente, o cenário atual se mostra complexo por diversos fatores, dentre os quais se destaca o perfil dos estudantes que chegam ao ensino superior. Com a chegada da chamada Geração Y (nascidos após 1979) às universidades, a forma de ensino-aprendizagem recebeu um duro golpe por parte dos novos alunos-clientes. Para esse público, nascido na época da Internet e com suas atividades ocorrendo em ritmo diferenciado, como se a vida fosse um videoclipe, a aprendizagem deve incluir, necessariamente, o uso de novas tecnologias, acesso às redes sociais virtuais, com aulas mais dinâmicas e conteúdo inovador.

Do outro lado da cena, na sala de aula, em que prevalece uma geração de professores migrando para o uso do computador, o desafio de tornar a aula mais atrativa e atualizada para esse público é um fantasma acadêmico que paira na classe. Educadores em Saúde agora possuem a capacidade para ministrar cursos a distância, desenvolver programação interativa eletrônica, participar de discussões em tempo real por meio de videoconferência e participar em atividades conexas destinadas a preparar os profissionais de educação em Saúde e promover a Saúde entre a população. A inclusão dessas novas tecnologias em instituições de ensino promove com mais facilidade as trocas de informação entre indivíduos. Essas se tornam mais acessíveis, os educadores deixam de ser o mestre que retém todo o conhecimento e os materiais pedagógicos excedem os livros-textos para tornarem-se programas e projetos mais extensos. A capacitação do educando para docência em novas tecnologias carece de uma nova configuração do processo didático e metodológico para uma formação concreta e propostas inovadoras ${ }^{5}$.

A mudança do paradigma se dá com a crescente digitalização do saber, a criação de portais de pesquisa e a ampliação de possibilidades de busca de conteúdo por parte de alunos e também de professores. Com o surgimento das redes sociais virtuais, os alunos permanecem conectados em comunidades além do horário de aula. Muitos encontram ali - com o apoio dos docentes - no espaço de grupos fechados, criados para este fim, uma continuação da sala de aula. Os professores que acompanham o progresso tecnológico já perceberam que, em vez de ver a tecnologia como uma ameaça, podem tê-la como aliada no campo do ensino e aprendizagem.

Observadores do emprego de novas tecnologias nas escolas e salas de aula há muito têm notado a relativamente modesta aplicação de maneira sistemática e integrada aos currículos. Embora a vida pessoal dos estudantes e professores tenha "evoluído" para a inclusão de mais e mais tecnologias, a situação educacional representa um paradoxo. Mesmo após inúmeros programas governamentais no Brasil desde a década de $1980^{6}$, a eficiente inclusão das tecnologias digitais, associadas a computadores, softwares específicos, meios digitais de comunicação, formação de pessoal, seja docente ou técnico, enfim todo o aparato técnico e humano, não produziu a disseminação nem o aumento qualitativo objetivado.

Diversas pesquisas de âmbito nacional realizadas nos Estados Unidos entre os anos de 2005 e 2007 apontaram para o aumento do emprego das tecnologias no trabalho docente em atividades complementares com aumento de produtividade, mas relativamente baixa utilização direta nos processos de ensino com foco no desenvolvimento dos estudantes ${ }^{7}$.

Docentes e estudantes utilizam as tecnologias mais frequentemente fora da escola do que durante as aulas propriamente ditas. Indubitavelmente, não se pode generalizar, pois há inúmeros profissionais que as empregam de forma inovadora, contudo é certamente exceção à regra, infelizmente.

Outro aspecto importante a se ressaltar nesse contexto é o conhecimento, por meio dos estudos na área das relações humanas, de que as pessoas tendem a não valorizar as "coisas" que possuem há longo tempo. Por exemplo, para a geração dos Baby Boomers (atualmente na faixa entre 50 e 60 anos), possuir eletricidade é 
algo absolutamente normal, porque sempre a tiveram. Agora, para as gerações mais novas, hoje, o que eles pensam ser normal? Vemos crianças pequenas já com seus próprios celulares nas mãos; eles têm acesso a essa tecnologia e pensam ser normal possuí-la. Inclusive, têm dificuldade em compreender como se vivia no passado sem os celulares e a Internet.

Os estudantes que ingressam atualmente no ensino superior possuem e-mail, smartphones, acesso aos recursos da Web (World Wide Web), mensagens de texto e todo um conjunto de dispositivos e aplicativos digitais que facilitam muitas de suas atividades cotidianas; para eles, tudo isso é normal. Dados recentes da Agência Nacional de Telecomunicações (ANATEL) informam que há mais celulares no Brasil do que pessoas! Em abril de 2013, contabilizavam-se 261 milhões de aparelhos, com crescimento de $8 \%$ em relação ao ano anterior. Além disso, não apenas possuir tecnologia é normal para eles, como vivenciar as mudanças tecnológicas cada vez mais presentes no seu dia a dia.

Em termos da abrangência das tecnologias digitais no cotidiano das pessoas e decorrentes influências na Educação, os motores de busca dos sites Google e Yahoo! têm recebido mais de 14 bilhões de acessos anuais, mais de 200 bilhões de e-mails são enviados todos os dias e se estimam mais de 2,3 trilhões de mensagens de texto diárias. As implicações diretas desse cenário são as várias opções e facilidades comunicacionais à disposição dos estudantes, o que torna a comunicação face a face na sala de aula apenas mais uma forma de se conectar e, talvez, a menos produtiva, intelectualmente falando ${ }^{1}$. Além da questão comunicacional, outro importante fator entra em cena, a saber, a produção de conteúdo pelo usuário (user-generated content) viabilizada pelas novas ferramentas de interação da Web 2.0. Destacam-se aqui as redes sociais virtuais (como Twitter, LinkedIn), as mídias sociais (Facebook, Google+), os blogs, mapeamento em tempo real, os podcasts, a produção e distribuição de vídeos (inclusive em tempo real, com streaming), etc., dentre uma variedade de plataformas, inclusive para dispositivos móveis, permitindo a produção e o compartilhamento dos mais diversos tipos de conteúdos. Certamente, a Educação tem à frente enorme desafio no sentido de se apropriar dessas tecnologias e as potencializar nos processos de ensino e aprendizagem.

Segundo o modelo de difusão das inovações de Everett Rogers ${ }^{8}$, parece que poucas tecnologias conseguiram cruzar a barreira de adoção eficientemente na área educacional, desde o momento de seu surgimento e apropriação pelos adotantes, diante da grande resistência e do conservadorismo dos professores. Talvez essas as barreiras na adoção correta e bem planejada das tecnologias digitais no ambiente escolar possam explicar resultados como os obtidos por Dwyer et al. ${ }^{9}$, em que não se observou correlação positiva direta entre a existência de recursos computacionais nas escolas e o desempenho dos alunos. As tecnologias não dispensam o professor, muito pelo contrário, continua em suas mãos a habilidade de integrar os recursos disponíveis na busca incansável pelo crescimento intelectual dos aprendizes ${ }^{6}$.
Nesta análise, o enfoque foi direcionado para duas destacadas e polêmicas tecnologias, a saber, as redes sociais virtuais e a educação a distância. Recuero ${ }^{10}$ define redes sociais como interações que acontecem entre as pessoas e derivam em grupos sociais, conectados por interesses afins. Ela ressalta que, para entender como funcionam e derivam os laços sociais, é necessário observar o todo como um sistema no qual os indivíduos estão ligados e suas ações afetam o resultado final. Em se tratando de redes sociais na Internet existem dois componentes dessa relação que possibilitam o funcionamento do sistema: os atores e as conexões. A autora ainda complementa: "Rede social é gente, é interação, é troca social. É um grupo de pessoas, compreendido através de uma metáfora de estrutura, a estrutura da rede". Segundo Mondini et al. ${ }^{11}$, as redes sociais digitais vêm modificando as formas de comunicação e relacionamento das pessoas, afetando, com isso, também as instituições de ensino (IESs).

Um trabalho das pesquisadoras da Universidade de Chicago (EUA) Saarinen et al. ${ }^{12}$ referiu-se a uma apresentação realizada na Academic Internal Medicine Week 2010 e abordava o uso das mídias sociais na Educação médica. As autoras dividiram e conceituaram mídias sociais e entenderam que o social está relacionado da seguinte forma: social significa pessoas, logo, os alunos são as pessoas, os técnicos de laboratório são pessoas, os pacientes são pessoas e a comunidade do campus é composta por pessoas. Mídia significa meio, logo, informações. Para as autoras, poderíamos criar e usar a mídia: fotos, vídeo, áudio, documentos, websites, tutoriais, mapas, revisões, links da Web, feeds de notícias, calendários de atividade, etc. As pesquisadoras exemplificam como usar as novas tecnologias na área da Saúde com o uso de Twitter, wikis, podcasting e blogs.

Diversos autores têm se debruçado sobre a questão do impacto do uso de redes sociais por alunos e professores. Shah et al. ${ }^{13}$ buscaram identificar benefícios do uso de mídias sociais virtuais no ganho de capital social e verificar o impacto na performance acadêmica dos pesquisados. Magro et al. ${ }^{14}$ tinham como objetivo verificar com estudantes universitários americanos como o Facebook poderia ser utilizado como ferramenta pedagógica. $\mathrm{Hew}^{15}$ se propôs a examinar como alunos fazem uso do Facebook para engajar a sala de aula em atividades colaborativas e mostrar como essa rede pode ser utilizada como ferramenta informal para organizar experiências em sala e explorar os fatores que promovem esse tipo de uso.

Choi $^{16}$ estudou experiências de implementação do Facebook em grupos para promover a colaboração dos estudantes; Barczyk e Duncan ${ }^{17}$ buscaram determinar a atividade de alunos e percepções sobre cursos em que o Facebook tem sido incorporado. Com foco os docentes, Chatterjee ${ }^{18}$ propôs estudar os antecedentes e as consequências da utilização da mídia social por professores universitários. Roblyer et al. ${ }^{19}$ procuram identificar as diferenças na adoção do Facebook por estudantes e docentes da faculdade do ponto de vista pessoal e educacional. Bicen e Uzunboylu ${ }^{20}$ tentaram descobrir como o Facebook e a Web 2.0 criavam um efeito 
positivo, quando usados na Educação, para investigar a opinião dos professores sobre o ambiente de aprendizado on-line.

Contextualizando um pouco mais a problemática enfocada nessa reflexão, o Brasil foi o país que mais cresceu em número de usuários no Facebook em 2011. O país saltou de 8,8 milhões de usuários em dezembro de 2010 para mais de 35 milhões no mesmo mês de 2011 , um crescimento de $298 \%$. O país fica na $4^{\text {a }}$ colocação em número de usuários da rede social, perdendo para os Estados Unidos, que possui 157 milhões de internautas, Indonésia, com 41,7 milhões, e Índia, com 41,3 milhões. Abaixo do Brasil ficaram México (30,98 milhões), Turquia (30,96 milhões), Reino Unido (30,4 milhões) e Filipinas (27 milhões). No total, o Facebook possui mais de 800 milhões de usuários ${ }^{21}$.

O Facebook é um site que permite aos usuários interagir e colaborar dentro de uma comunidade virtual predefinido. Muitas vezes chamado de site de rede social, o Facebook é uma ferramenta de comunicação e colaboração on-line que permite aos usuários construir um perfil público ou privado, a fim de se conectar e interagir com as pessoas que fazem parte da sua rede social virtual alargada ${ }^{22}$. O Facebook tem como principal missão "tornar o mundo mais aberto e conectado. As pessoas usam o Facebook para ficar conectadas com amigos e familiares, para descobrir o que está acontecendo no mundo e para compartilhar e expressar o que importa para eles"23. O Facebook foi fundado em fevereiro de 2004 para ajudar as pessoas a se comunicarem de forma mais eficiente com seus amigos, familiares e colegas de trabalho. A empresa desenvolveu tecnologias que facilitaram o compartilhamento de informações por meio de interações sociais mediadas digitalmente e que visam estender ao mundo virtual as relações sociais presenciais. Inicialmente, o Facebook era apenas para estudantes universitários, mas atualmente é aberto para que todos possam se inscrever e compartilhar suas informações ${ }^{24}$.

No Brasil, o Facebook ascendeu como um forte líder da categoria das redes sociais, com quase 44 milhões de visitantes únicos em dezembro de 2012, 22\% a mais que no ano anterior ${ }^{25}$. De acordo com Spers et al. ${ }^{26}$, projeta-se para 2020 uma penetração da Internet nos domicílios brasileiros de 95 a 100\% na classe A, de 48 a $70 \%$ na classe B e de 10 a 50\% nas classes D e E. Em se tratando de conexões com maior velocidade, $99 \%$ das conexões de Internet serão do tipo banda larga para a classe A, 90\% para a classe B, 60\% para a classe C e $25 \%$ para as classes D e E.

$\mathrm{Na}$ área da Saúde, a aplicabilidade dessa tecnologia, em termos educacionais, segue o mesmo roteiro presente entre os profissionais da Educação, destacando-se o uso pessoal, informal, nem tanto a extração das possibilidades educativas. Em trabalho realizado em 2007, Thompson et al. ${ }^{27}$ buscaram identificar a frequência e o conteúdo das redes sociais on-line entre estudantes de Medicina e residentes, tendo como sujeitos de pesquisa 501 estudantes de Medicina e 312 residentes da Universidade da Flórida e como objeto de pesquisa os perfis do Facebook desse público. O resultado mostrou que o uso do Facebook é comum entre os médicos estagiários, com $44,5 \%$ dos pesquisados tendo uma conta. Estudantes de medicina usam com frequência a rede $(64,3 \%)$ e residentes com menos frequência. Quanto maior tempo de estudo/formação, menor o uso do Facebook. O estudo sugeriu que enquanto o uso das redes sociais por médicos estagiários é comum na cultura atual de profissionais emergentes, a maioria dos usuários permite que qualquer um veja seu perfil, havendo necessidade de profissionalismo, com atenção às identidades pessoais e profissionais.

Pettit $^{28}$ apontou para as implicações das tecnologias da informação para a educação profissional em Saúde. O artigo explorou os benefícios e as barreiras do progresso tecnológico e coloca questões fundamentais sobre as informações tecnológicas para a educação profissional. O autor encoraja os educadores em Saúde a facilitarem seu discurso sobre seus papéis e suas responsabilidades em relação ao avanço tecnológico. Também em 2008, Joly ${ }^{29}$ relatou que, em novembro de 2007, o Facebook lançou uma série de novos recursos, incluindo páginas que permitem a colegas, universidades e outras escolas criar um perfil e recrutar fãs entre usuários da rede. Até então, instituições como organizações, grandes companhias ou mesmo pequenos negócios não eram realmente bem-vindos. $\mathrm{O}$ artigo apresentou histórias de sucesso no uso de aplicativos do Facebook por várias instituições de ensino, entre elas: Standford University, Allegheny College e Butler University.

Em um dos trabalhos nacionais na área da Psicologia, Marcos e Vizzotto ${ }^{30}$ buscaram identificar o impacto do uso da Internet em estudantes do segmento da Saúde. A fim de investigar a dependência do uso da Internet, as autoras realizaram pesquisa com 150 alunos utilizando como parâmetro os critérios diagnósticos de distúrbios para jogos patológicos e modificando-os para caracterizar a dependência do uso da Internet. O estudo mostrou que a dependência da Internet vai além do tempo de conexão. Outras características que, segundo o estudo, auxiliam a diagnosticar o uso compulsivo da Internet são: o tipo de uso, o uso repetitivo e pouco criativo, como a pessoa se sente quando não está conectada à Internet (ansiosa, triste, irritada e apreensiva para acessar novamente a Internet) e como está a vida presencial dessa pessoa (se mantém encontros sociais e amorosos, trabalho, escola, lazer, também fora na Internet). Os resultados alertaram que o número de internautas que realizam o uso patológico da Internet mostrava-se bastante relevante $(57 \%)$ e confirmaram que a patologia se instala quando o indivíduo restringe seu mundo ao virtual, não transpondo para o mundo real, utilizando, assim, a Internet em detrimento de outras atividades.

Além das mídias sociais, grande impacto tem sido observado pelas aplicações da Educação a Distância (EaD), que também cria polêmicas entre os docentes, especialmente na área da Saúde. Para este trabalho, considerou-se EaD quando a informação e o aprendiz estão separados pelo tempo, pelo espaço, ou por ambos. As observações sobre o público aderente aos estudos na modalidade em $\mathrm{EaD}$ mostram que, usualmente, são pessoas mais maduras (acima dos 25 anos de idade), empregadas 
e que possuem automotivação, disciplina e organização para o estudo independente.

Conforme postula o professor Qureshi ${ }^{31}$, a modalidade em $\mathrm{EaD}$ está substituindo a modalidade de educação face a face, pois se adéqua no formato "centrado no estudante" com o suporte de ferramentas e interações para que o estudante percorra trilhas de aprendizagem que o conduzem ao desenvolvimento de competências e habilidades objetivadas. Os conteúdos devem focalizar cenários reais do cotidiano e resolução de problemas práticos por intermédio da intervenção de tutores, do trabalho colaborativo e com interações sincrônicas ou assíncronas.

Algumas vantagens têm sido apontadas com o estudo em $\mathrm{EaD}$, como a possibilidade de os estudantes investirem mais esforço nos itens de sua maior dificuldade, eles podem escolher os melhores momentos para estudar, seja durante transporte, seja em intervalos de refeições. Obviamente, os estudantes devem respeitar os prazos estabelecidos para cada uma das atividades propostas. A modalidade via EaD oportuniza a estudantes de regiões distantes dos grandes centros o acesso a especialistas importantes de cada área e a materiais didáticos atualizados, bem como a vídeos e simuladores, recursos esses antes apenas disponíveis para alunos de cursos presenciais nas grandes faculdades. Finalmente, os custos de cursos na modalidade em EaD tendem a ser mais baixos, pela maior escala de alunos participantes.

Por outro lado, uma das maiores dificuldades dessa modalidade de educação é o fator motivacional, pois o estudo independente, sem o contato pessoal direto, leva ao desestímulo e ao fracasso nos rendimentos. Entende-se que ainda hoje nem todas as pessoas estão preparadas para essa modalidade, cuja dificuldade tende a ser diretamente proporcional aos anos de estudo em salas de aula presenciais.

Contudo, o cenário está mudando, especialmente no Brasil, pois os dados divulgados pelo Ministério da Educação sobre os resultados do Exame Nacional de Desempenho de Estudantes (ENADE) apontam para melhor desempenho acadêmico dos estudantes advindos de cursos EaD, na maioria das áreas sob avaliação, quando comparados aos mesmos cursos presenciais. Ressalta-se, ainda, que algumas associações de classe profissionais conseguiram obstaculizar os cursos em EaD em suas áreas, como os advogados e os médicos.

\section{CONSIDERAÇÕES FINAIS}

Pelo que pudemos verificar o caminho para adoção de novas tecnologias em sala de aula está fortemente indicado em razão do público que adentra as salas: conectado, usuário de tecnologia como nova forma de aprendizado. Querer negar a necessidade de mudanças por parte dos docentes é querer oferecer um produto - no caso em questão, o conhecimento - a um aluno-cliente que está cada vez mais exigente.

É claro que apenas informatizar a forma de transmitir o conteúdo não garante, em si, a aprendizagem. Esta se dá quando o ensino faz sentido a quem quer aprender. Nesse caso, a tecnologia é apenas um novo meio pelo qual o ensino pode tornar-se aprendizado.

Da aula GLS (giz, lousa e saliva) ao novo modelo consumido pelas Gerações X e Y, muitas mudanças tiveram lugar, dentro e fora da sala de aula. Com o conhecimento centralizado no docente $\mathrm{e}$ na literatura por ele oferecida para o novo modelo em que o professor passa de detentor do saber para facilitador, indicando o que, como e onde obter informações de qualidade para os alunos, muitos ficaram pelo caminho. É natural que a adoção de novas tecnologias demande processos de acomodação e adaptações e que devem ser elaboradas para ambos os públicos: docentes e discentes. Sendo assim, introduzir e adotar tecnologia na sala de aula trata-se de processo que nem sempre vem na velocidade desejada.

Especificamente para os estudantes da área da Saúde, as notícias são bastante positivas, haja vista que o próprio mercado de trabalho sinaliza com a adoção cada vez mais intensa da tecnologia nos consultórios, seja nos equipamentos, na forma de registro de dados de pacientes, bem como na nova forma de relacionamento entre pacientes e profissionais de Saúde. Se o uso da tecnologia já começar dentro da sala de aula, a continuidade da adoção das novas formas de acesso à informação continuará se dando de forma natural.

$\mathrm{O}$ alerta maior fica para o segmento dos docentes, que assimilou de uma forma o conteúdo que hoje se transmite tecnologicamente. Fazer a ponte entre o saber adquirido no passado e a forma como didaticamente deve ser transmitido - com o uso das novas tecnologias - a esse novo público conectado não é tarefa das mais fáceis. Querer mudar, aprender e adaptar são etapas que poderão garantir a continuidade do sucesso na profissão. Insistir em velhas formas para um novo público poderá indicar o caminho do fracasso na profissão. A opção da mudança, da adoção do long life learning, fica a critério de cada um.

\section{REFERÊNCIAS}

1. Kittleson MJ. The future of technology in health education: challenging the traditional delivery dogma. Am J Health Educ. 2009;40(6):310-6.

2. Rüdiger F. Introdução às teorias da cibercultura: perspectivas do pensamento tecnológico contemporâneo. Porto Alegre: Editora Sulina; 2004

3. Araújo KTM, Goulart EE. Um estudo sobre a utilização das TIC's na região de Santo André. Rev Iberoamericana Educación. 2006;38(3):1-10
4. Negroponte N. A vida digital. São Paulo: Companhia das Letras; 1995

5. Ribas D. A docência no ensino superior e as novas tecnologias Disponível em: http://web03.unicentro.br/especializacao/Revista Pos/P\%C3\%A1ginas/3\%20Edi\%C3\%A7\%C3\%A3o/Humanas/ PDF/3-Ed3_CH-DocenciaEns.pdf. Acesso em 1 jul. 2015.

6. Cardoso AM, Azevedo JF, Martins RX. Histórico e tendências da aplicação das tecnologias no sistema educacional brasileiro. Rev Digital CVA. 2013;8(30):1-11 
7. Means B. Tedchnology and education change: focus on student learning. J Res Tec Educ. 2010;42(3):285-307.

8. Rogers EM. Diffusions of Innovations. $5^{\text {th }}$ ed. New York: The Free Press; 2003

9. Dwyer T, Wainer J, DutraRS, Covic A, Magalhães VB, Ferreira LRR, et al. Desvendando mitos: os computadores e o desempenho no sistema escolar. Educ Soc. 2007;28(101):1303-28. http://dx.doi.org/10.1590/S0101-73302007000400003

10. Recuero R. Redes sociais na Internet. Porto Alegre: Editora Sulina; 2009

11. Mondini LC. Redes sociais digitais: uma análise de utilização pelas instituições de ensino superior do sistema ACAFE de Santa Catarina. Rev Eletrônica Ciênc Adm. 2012;11(1):48-60.

12. Saarinen C, Arora V, Ferguson B, Chretien K. Incorporating Social Media into Medical Education. Acad Int Med Week Insight. 2011;9(1):12-3

13. Shah V, Subramanian S, Rouis S, Limayem M. A Study on the Impact of Facebook Usage on Student's Social Capital and Academic Performance. AMCIS. 2012;27.

14. Magro M, Ryan K, Ryan S, Sharp J. Facebook's use in higher education. AMCIS. 2012;10

15. Hew KF. Student's and teachers' use of Facebook. Comp Hum Behavior. 2011;27(2):662-76. http://dx.doi.org/10.1016/j.chb.2010.11.020

16. Choi A. Use of Facebook Group Feature to Promote Student Collaboration. Macon, GA: American Society for Engineering Education; 2013

17. Barczyk CC, Duncan DG. Facebook in higher education courses: an analysis of students' attitudes, community of practice, and classroom community. Int Business Manag. 2013;6(1):1-11. http://dx.doi.org/10.3968\%2Fj.ibm.1923842820130601.1165

18. Chatterjee S. Facebook as a Tool in Higher Education and the Gender Issue: a Survey among Students in Bankura. Bhatter College J Multidisc Studies. 2012;89-94.

19. Roblyer MD, McDaniel M, Webb M, Herman J, Witty JV. Findings on Facebook in higher education: A comparison of college faculty and student uses and perceptions of social networking sites. Internet Higher Educ. 2010;13(3):134-40. http://dx.doi.org/10.1016/j.iheduc.2010.03.002

20. Bicen $H$, Uzunboylu $H$. The use of social networking sites in education: a case study of Facebook.. J Universal Comp Science. 2013;19(5):658-71. http://dx.doi.org/10.3217/jucs-019-05-0658
21. Portal G1. Número de usuários brasileiros no Facebook cresce 298\% em 2011. Disponível em: http://g1.globo.com/tecnologia/noticia/2012/01/ umero-de-usuarios-brasileiros-no-facebook-cresce-298-em-2011. html. Acesso em: 01 jul. 2015.

22. Irwin CG, Ball LE, Desbrow B, leveritt M. Students' perceptions of using Facebook as an interactive learning resource at university. Australasian J Educ Technology. 2012;28(7):1221-32

23. Esteves KK. Exploring Facebook to enhance learning and student engagement: a case from the University of Philippines (UP) open University. Malaysian J Distance Educ. 2012;14(1):1-15

24. Babb TA. Counselor education student perceptions of the american counseling association ethical code as it pertains to the use of Facebook. Dissertação (Mestrado). Toledo University, Curso de Educação. Toledo (EUA): 2012.

25. ComScore. ComScore Releases '2013 Brazil digital future in Focus' report. Disponível em: http://www.comscore.com/Insights/Press-Releases/2013/3/ comScore-Releases-2013-Brazil-Digital-Future-in-Focus-Report. Acessso em: 1 jul. 2015.

26. Spers RG, Wright JTC, Silva ATB. Internet Banda Larga: um estudo prospectivo exploratório sobre a sua penetração, tecnologias de conexão e impactos no Brasil em 2020. Rev Adm Inovação. 2012;9(2):55-78

http://dx.doi.org/10.5773/rai.v9i2.675

27. Thompson LA, Dawson K, Ferdig R, Black EW, Boyer J, Coutts $J$, et al. The intersection of online social networking with medical professionalism. J Gen Intern Med. 2008;23(7):954-7. http://dx.doi.org/10.1007/s11606-008-0538-8

28. Pettit ML. Implications of information technology for the health education profession. Am J Health Studies. 2008;23(1):41.

29. Joly K. The Facebook applications: the game changer. Disponível em: http://www.universitybusiness.com/article/ facebook-applications-game-changer. Acesso em: 1 jul. 2015.

30. Marcos VP, Vizzotto MM. Uso patológico da internet em estudantes universitários. In: II Congresso Luso-Brasileiro de Psicologia da Saúde/l Congresso Ibero-Americano de Psicologia da Saúde, 2011, São Bernardo do Campo. Transformações socioculturais e promoção de saúde. São Bernardo do Campo, 2011.

31. Qureshi AU. Online distance learning: a modle of short communication for modern day higher education. Int $\mathrm{J}$ Health Educ. 2012;134-43. 